\title{
Theory of Shape-Shifting Droplets
}

\author{
Pierre A. Haas, ${ }^{1}$ Raymond E. Goldstein, ${ }^{1, *}$ Stoyan K. Smoukov, ${ }^{2, \dagger}$ Diana Cholakova, ${ }^{3}$ and Nikolai Denkov ${ }^{3}$ \\ ${ }^{1}$ Department of Applied Mathematics and Theoretical Physics, Centre for Mathematical Sciences, \\ University of Cambridge, Wilberforce Road, Cambridge CB3 OWA, United Kingdom \\ ${ }^{2}$ Department of Materials Science and Metallurgy, University of Cambridge, \\ 27 Charles Babbage Road, Cambridge CB3 OFS, United Kingdom \\ ${ }^{3}$ Department of Chemical and Pharmaceutical Engineering, Faculty of Chemistry and Pharmacy, \\ University of Sofia, 1164 Sofia, Bulgaria \\ (Received 2 September 2016; published 21 February 2017)
}

\begin{abstract}
Recent studies of cooled oil emulsion droplets uncovered transformations into a host of flattened shapes with straight edges and sharp corners, driven by a partial phase transition of the bulk liquid phase. Here, we explore theoretically the simplest geometric competition between this phase transition and surface tension in planar polygons and recover the observed sequence of shapes and their statistics in qualitative agreement with experiments. Extending the model to capture some of the three-dimensional structure of the droplets, we analyze the evolution of protrusions sprouting from the vertices of the platelets and the topological transition of a puncturing planar polygon.
\end{abstract}

DOI: 10.1103/PhysRevLett.118.088001

Shape generating chemical systems are very literal instantiations of Turing's quest for the chemical basis of morphogenesis [1]. Since they are to living systems what the shadows perceived by the eponymous cavemen of Plato's allegory [2] are to reality, it is the apparent simplicity of these systems that makes them ideal candidates for untangling the physical underpinnings of morphogenesis. A promising exemplar of such systems, revealed in recent work by Denkov et al. [3], is small, micron-sized oil droplets suspended in an aqueous surfactant solution: as the temperature of the solution is slowly lowered towards the bulk freezing point of the oil, the initially spherical droplets undergo remarkable shape transformations. The droplets flatten, and evolve through a sequence of polygonal shapes to first become hexagons, and later triangles or quadrilaterals [Fig. 1(a)]. At later stages, droplets grow thin protrusions, before ultimately thinning out into filaments.

This host of deformations was attributed to freezing of the surfactant layer before the droplet bulk, upon which the formation of a plastic rotator phase [4] of self-assembled oil molecules next to the drop surface becomes energetically favorable [Fig. 1(b)]. This rotator phase, characterized by long-range translational order, rearranges into a frame of plastic rods at the drop perimeter which supports the polygonal structure [Fig. 1(c)]. Simple energy arguments [3] were used to show that it is this rotator phase that must provide the energy to overcome the surface tension of the droplets and deform them. Other work [5] contends that

Published by the American Physical Society under the terms of the Creative Commons Attribution 3.0 License. Further distribution of this work must maintain attribution to the author(s) and the published article's title, journal citation, and DOI. these and similar deformations observed in related systems are the result of surface monolayer freezing only, however, and this was further discussed in a series of very recent papers [6-8]. The picture that emerges from this analysis is that the initial deformations of the spherical droplets to polyhedral shapes may be driven by the topological defects and the associated elastic scalings $[9,10]$ resulting from the freezing of a planar, multi- or monomolecular layer, before the formation of tubules of rotator phase stretches and flattens the droplets. This expansion of the rotator phase drives the transition between the different polygonal stages as well as the later formation of protrusions, but the

(a)

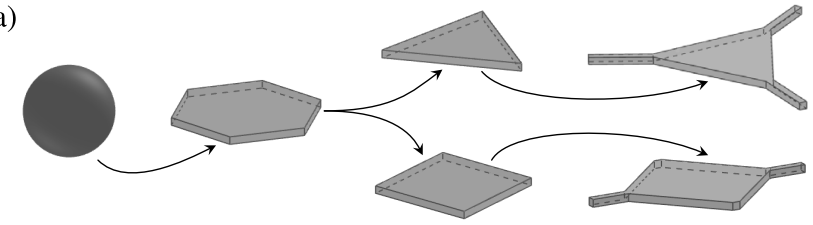

(b) $\triangle$

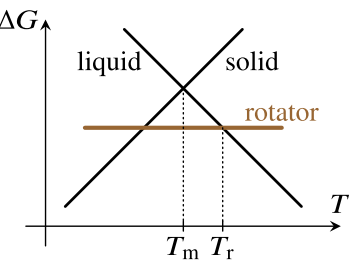

(c)

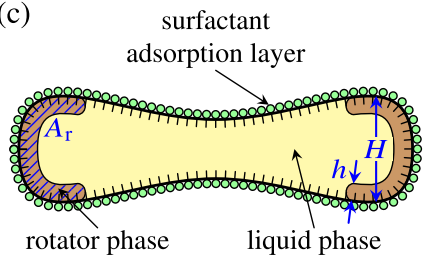

FIG. 1. Shape-shifting droplets. (a) Main stages of droplet shape evolution, following Ref. [3]. Initially spherical droplets flatten, first into hexagons, then into quadrilaterals or triangles, before growing thin protrusions. (b) Energy diagram, showing a range of temperatures close to the melting temperature where the formation of a rotator phase is energetically favored. (c) Cross section of flattened droplet. A layer of plastic rotator phase has formed at the edges of the droplet. 
underlying geometric and energetic balances have not yet been spelled out.

Here, we present the first theoretical description of this process: we analyze the competition between this formation of the rotator phase and surface tension which resists the deformations. Successive geometric approximations at different levels elucidate the dynamics of these polygonal droplets. Our starting point is the experimental observation that the edges of the polygonal droplets are nearly straight, suggestive of a high energy cost to bend, and thus an even higher cost to stretch the rotator phase. We therefore assume the rotator phase to form straight, rigid rods at this point, but relax this assumption in later parts of the analysis. Vertices of the polygonal droplets correspond to defects in the rotator phase. A very large majority of the interior angles of the polygons are seen in experiments to have measures close to $60^{\circ}$ or $120^{\circ}$, suggesting that the defect energy landscape has strong minima near these values, which we therefore assume to be the only possible interior angles. Let $u, v$ denote, respectively, the number of vertices with angles of $60^{\circ}$ and $120^{\circ}$, so that if the polygons are convex,

$$
60^{\circ} \times u+120^{\circ} \times v=180^{\circ} \times(u+v-2) .
$$

The resulting equation, $2 u+v=6$, has four solutions over the non-negative integers:

$$
(u, v)=(0,6),(1,4),(2,2),(3,0) .
$$

There are five polygonal shapes satisfying these requirements, shown in Figs. 2(a) and 2(b): equiangular hexagons, pentagons, two types of quadrilaterals (isosceles trapezoids and parallelograms), and equilateral triangles. These shapes have variable aspect ratios, and by contracting one of the sides to zero while extending the neighboring sides, hexagons can evolve into pentagons, and pentagons can evolve into either trapezoids or parallelograms. Of the latter two, only the trapezoids can evolve into triangles. This recovers the experimental sequence of shapes and, in particular, the observed dichotomy between triangles and quadrilaterals as final outcomes [Fig. 1(a)].

The discrete defect energy has thus constrained the analysis of possible shapes, but does not otherwise affect the shape dynamics. At the next level of the description, we therefore turn to the energetics of the droplets. Let $V$ be the droplet volume, assumed fixed, and $S$ its curved surface area, and assume that $V$ is the sum of the volumes of the rotator and liquid phases, $V=V_{r}+V_{l}$, and that their densities are equal. With $\mu_{r}$ and $\mu_{l}$ the chemical potentials per unit volume of the rotator and liquid phases, the energy of the droplet is

$$
E=\mu_{l} V_{l}+\mu_{r} V_{r}+\gamma S,
$$

where $\gamma \approx 5 \mathrm{mN} / \mathrm{m}$ is the (constant) surface tension [7,11]. For the phase change to the rotator phase to be energetically favorable, $\Delta \mu \equiv \mu_{l}-\mu_{r}>0$. At the temperature $T_{r}$ of the liquid-to-rotator phase transition [Fig. 1(b)], the two phases are in equilibrium, with $\Delta \mu=0$. When the system is cooled below $T_{r}, \Delta \mu=\Delta S_{l r}\left(T_{r}-T\right)$, where $\Delta S_{l r} \approx 6 \times 10^{5} \mathrm{~N} / \mathrm{m}^{2} \mathrm{~K}$ is the entropy of transition per unit volume from the liquid to the rotator phase [12] and $T_{r}-T \approx 1 \mathrm{~K}$ during the platelet stage is sufficient to overcome the energy cost associated with the expanding droplet surface. Next, we approximate $V_{r}=A_{r} P$, where $P$ is the perimeter of the polygonal frame and $A_{r}$ is a characteristic cross-sectional area of the rotator phase [Fig. 1(c)], depending on the intrinsic properties of the oil and the surfactant. Approximating the cross section of the rotator phase as a semicircular ring of outer diameter $H \approx 2 \mu \mathrm{m}$ and thickness $h \approx 100 \mathrm{~nm}$ [3,7] [Fig. 1(c)], we estimate $A_{r} \approx 0.3 \mu \mathrm{m}^{2}$.

Omitting constant terms and rescaling with the perimeter $P_{0}=\left(6 \pi^{2} V\right)^{1 / 3}$ of the initial spherical droplet, we obtain the dimensionless energy $\mathcal{E}=E / \gamma P_{0}^{2}$,

$$
\mathcal{E}=\mathcal{S}-\alpha \mathcal{P}, \quad \alpha=\frac{A_{r}}{V^{1 / 3}} \frac{\Delta \mu}{\left(6 \pi^{2}\right)^{1 / 3} \gamma},
$$

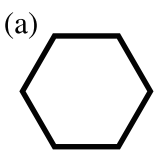

$(0,6)$ (b)

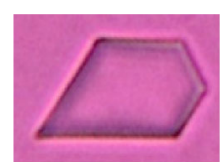

(c)

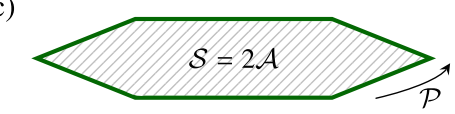

(d)

$(1,4)$

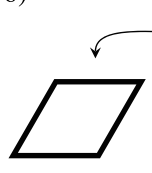

$(2,2)_{1}$
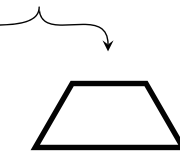

$(2,2)_{2}$

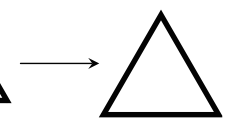

$(3,0)$

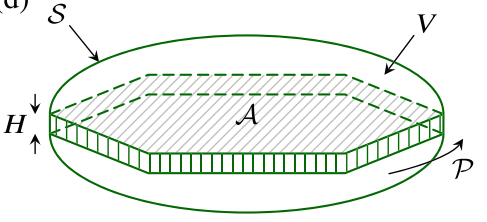

(e)

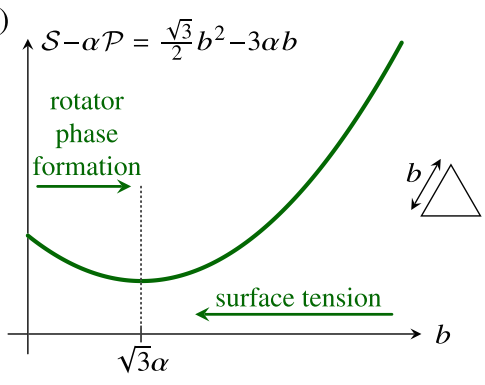

FIG. 2. Model of shape-shifting droplets. (a) Classification of polygonal shapes with interior angles of $60^{\circ}$ or $120^{\circ}$, and transitions between shapes. (b) Experimental image of pentagon state. (c) Simplest droplet model: a polygonal frame of area $\mathcal{A}$ and perimeter $\mathcal{P}$ defines a flat droplet of surface area $\mathcal{S}=2 \mathcal{A}$. (d) Extended droplet model: conservation of the droplet volume $V$ and the finite height $H$ of the rim of rotator phase modify the relation between $\mathcal{S}$ and $\mathcal{A}$. (e) Droplet energy: effect of competing surface tension and tendency to form rotator phase illustrated for the simplest, triangular droplet shape. 
where $\mathcal{S}=S / P_{0}^{2}$ and $\mathcal{P}=P / P_{0}$. This Gibbs-like energy results from a Legendre transform from a statistical ensemble of constant $\mathcal{P}$ to one where $\alpha$ is held fixed. The dimensionless $\alpha>0$ is the key parameter of our analysis. For initial droplet radii of 1-50 $\mu \mathrm{m}$ [7], we estimate $\alpha \approx 5.7-0.1$. In the experimental system, $\alpha$ varies because of the variation of $\Delta \mu$ with temperature (and thus with time), the variations of $A_{r}$ resulting from the kinetics of phase change, and, to a lesser extent, the weak variation of $\gamma$ with temperature [7]. Here, we shall assume $\alpha$ to be constant for simplicity, thereby averaging over this time variation.

The next step is to relate the surface area $\mathcal{S}$ to the geometry of the polygonal frame, which spans an area $\mathcal{A}$. In the simplest approximation, we consider a flat twodimensional droplet [Fig. 2(c)], for which $\mathcal{S}=2 \mathcal{A}$. This is the leading-order term of an expansion of $\mathcal{S}$ with corrections [14] arising from volume conservation and the thickness $H \approx 2 \mu \mathrm{m}$ [3] of the rim of rotator phase [Fig. 2(d)] that we shall consider later.

The competition captured by Eq. (4) between the rotator phase and surface tension is illustrated for equilateral triangles in Fig. 2(e), where $\mathcal{E}=\sqrt{3} b^{2} / 2-3 \alpha b$, with $b$ the edge length. Surface tension acts to decrease the side lengths of the triangle, which the formation of the rotator phase tends to expand. For the other shapes, disproportionation of edge lengths gives additional degrees of freedom, and we must derive the equations governing the shape dynamics to explore this aspect. We impose a standard constitutive law [13], that the normal velocity of the perimeter is proportional to the thermodynamic pressure resulting from the corresponding energy variations, and derive the corresponding equations in the Supplemental Material [14]. The time scale for these deformations is set by the effective viscosity $L$ of the droplets [14]. Videos showing the numerically computed evolution of hexagons into triangles, parallelograms, or trapezoids are available online [14]; whether a trapezoid evolves into a triangle or not depends on the aspect ratio of the droplet when it reaches the trapezoid state.
A linear stability analysis around the regular hexagonal shape [14] reveals three linearly independent growing modes: a triangle mode [Fig. 3(a), scaled eigenvalue 1] and two parallelogram modes [Fig. 3(b), scaled eigenvalue $2 / 3$. We take these eigenmodes as a basis for initial perturbations of prescribed magnitude $\varepsilon$ around the regular hexagon and map the perturbations yielding, respectively, triangles, parallelograms, or trapezoids onto the surface of a sphere [Fig. 3(c)]. Varying the parameters $\alpha$ (in the experimental range determined above) and $\varepsilon$, we compute the respective extents of these regions and thus the proportion of initial hexagons evolving into triangles and parallelograms [Figs. 3(d) and 3(e)]: at small $\varepsilon$, hexagons are overwhelmingly likely to evolve into triangles, but as $\varepsilon$ is increased, parallelograms and trapezoids dominate, with trapezoids somewhat less likely to arise than parallelograms. To relate this to experiments, we observe that $\varepsilon$ should increase with increasing cooling rate: at faster cooling, the arrangement of topological defects on the surface of the spherical droplet is less regular at the time when the rotator phase starts to form. Thus, the higher the cooling rate, the less likely droplets (of the same initial size) are to evolve into triangles, as observed in experiments [7]. Further, small variations in the positions of the topological defects on the surface are exacerbated by the small surface area of the smaller droplets, and thus $\varepsilon \propto V^{-2 / 3}$. Since $\alpha \propto V^{-1 / 3}$, it follows that, if all parameters other than $V$ are held constant, $\alpha \propto \varepsilon^{1 / 2}$. Hence, from Fig. 3(a), smaller droplets are less likely to evolve into triangles. This corresponds to the experimental observation [7] that small droplets are very likely to elongate into thin rods. In experiments [3] with $15 \mu \mathrm{m}$ droplets $(\alpha \approx 0.4)$ at a cooling rate of $0.2 \mathrm{~K} / \mathrm{min}$, about a quarter of the droplets evolved into triangles. From Fig. 3(a), we estimate $\varepsilon \approx 0.09$ for this cooling rate.

We now turn to the topological transition of droplet puncture: experimentally, some parallelograms elongate into thin rods, while others puncture in their center before

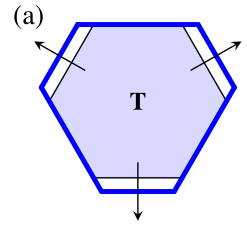

(b)

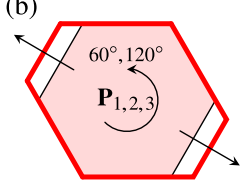

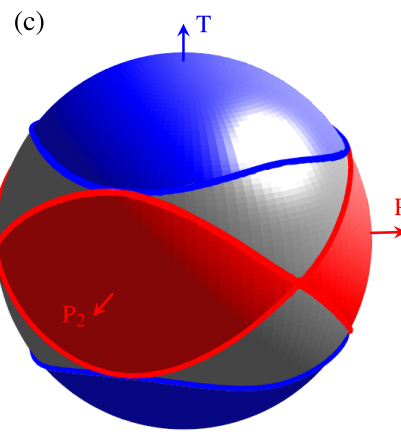
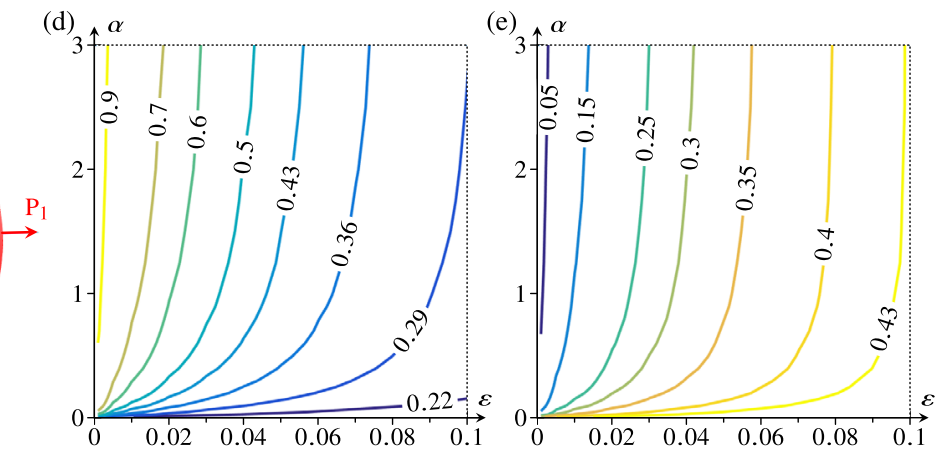

FIG. 3. Droplet modes and shape statistics. (a) Triangle mode (T), with eigenvalue 1. (b) Parallelogram mode (P), with eigenvalue $2 / 3$. There are two linearly independent modes of this ilk; others are obtained by rotation by $60^{\circ}$ or $120^{\circ}$. (c) Shape regions for perturbations of fixed magnitude $\varepsilon$ around the hexagon state, with eigendirections $\mathrm{T}$ and $\mathrm{P}$ indicated. Linearly independent $\mathrm{P}$ modes span the equatorial plane. Parameter values are $\alpha=1, \varepsilon=0.04$. (d) Proportion of initial hexagons that evolve into triangles in $(\varepsilon, \alpha)$ space. (e) Proportion of initial hexagons that evolve into parallelograms in $(\varepsilon, \alpha)$ space. 

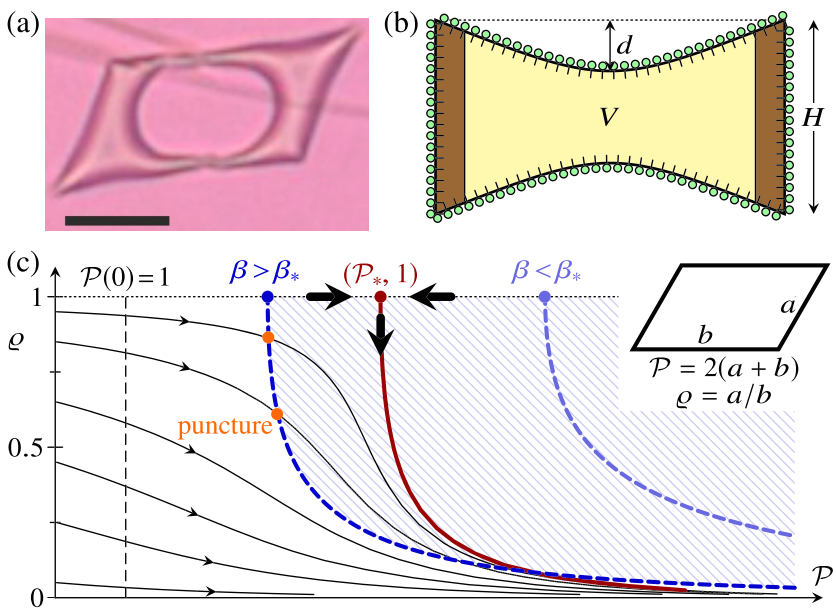

FIG. 4. Droplet puncture. (a) Punctured parallelogram as outcome of droplet shape evolution. Scale bar $20 \mu \mathrm{m}$. (b) Idealized cross section of an inverted droplet. (c) Trajectories of Eq. (6) indicated by solid lines, overlaid with the puncture condition Eq. (5), represented by dashed lines. Inset: Definition of geometric variables. Thick solid line shows trajectory through saddle. Parameter values are $\alpha=1, H / V^{1 / 3}=0.25,0.047$.

freezing [Fig. 4(a)], a strong experimental signature of the formation of rotator phase next to the perimeter [3]. A parallelogram with side lengths $a, b$ is most conveniently described in terms of its perimeter $\mathcal{P}=2(a+b)$ and aspect ratio $\varrho=a / b$ [Fig. 4(c), inset]. By symmetry, we may restrict the analysis to $0<\varrho \leq 1$. As the droplet expands, its surface inverts because of the finite thickness $H$ of the rim of rotator phase [Fig. 4(b)]. The drop punctures when, at a given aspect ratio, the depth $d$ of the inverted cap reaches the value $H / 2$. The condition $d \geq H / 2$ is equivalent to the droplet exceeding the critical perimeter [14],

$$
\mathcal{P}_{\text {crit }}(\varrho)=C \frac{V^{1 / 6}}{H^{1 / 2}} \frac{1+\varrho}{\sqrt{\varrho}} g(\varrho),
$$

where $C \approx 0.75$ is a numerical constant, and where $g(\varrho)$ is a decreasing function of $\varrho$, with $g(1)=1$, that must be evaluated numerically, although results do not change qualitatively if it is taken to be constant [14]. The evolution of $\mathcal{P}$ and $\varrho$ is governed by [14]

$$
\dot{\mathcal{P}}=8 \sqrt{3}\left(\frac{2 \alpha(1+\varrho)^{2}}{\sqrt{3} \mathcal{P} \varrho}-1\right), \quad \dot{\varrho}=-\frac{4 \sqrt{3}}{\mathcal{P}}\left(1-\varrho^{2}\right),
$$

which has a fixed point at $(\mathcal{P}, \varrho)=\left(\mathcal{P}_{*}, 1\right)$, with $\mathcal{P}_{*}=8 \alpha / \sqrt{3}$. Figure $4(\mathrm{c})$ shows a partial phase portrait of this dynamical system, overlaid with the puncture condition Eq. (5). The dynamics of the model thus allow the two types of outcome seen in experiments: if $\mathcal{P}_{\text {crit }}(1)>\mathcal{P}_{*}$, droplets cannot puncture and must elongate into thin rods, while, if $\mathcal{P}_{\text {crit }}(1)<\mathcal{P}_{*}$, droplets with large enough $Q$ must puncture [Fig. 4(c)]. The condition
$\mathcal{P}_{\text {crit }}(1)>\mathcal{P}_{*}$ rearranges to $\beta \equiv \alpha H^{1 / 2} / V^{1 / 6}<\beta_{*} \approx 0.33$. Thus, droplets with initial radius $R \gtrsim 7 \mu$ m cannot puncture; this value is borne out by experiments [7], although the largest droplets freeze before reaching these stages. Experiments, however, show that the smallest droplets very rapidly elongate into rods without puncture. This signals a breakdown of the assumption that $H$ is independent of $V$ : for small droplets, the extent of the rotator phase becomes comparable to the droplet size, and so the geometry and kinetics of the rotator phase may no longer be neglected. An analysis incorporating the intrinsic curvature of the rotator phase [Fig. 1(c)] leads to an explicit dependence of $H$ on droplet size [14] and could explain the observed behavior, but does not resolve its molecular origin. Further understanding of the kinetics of phase change must be gained to explain the size dependence of this transition.

Finally, we address the formation of protrusions sprouting from the vertices of the polygons. To this end, we must relax our initial assumption that the droplets have straight rigid sides, for otherwise the formation of a protrusion corresponds to replacing one defect with two defects (one of $120^{\circ}$ with two $120^{\circ}$ ones, or even one of $60^{\circ}$ with two $150^{\circ}$ ones), which is energetically unfavorable [16]. However, by the time protrusions appear in the experimental system, the sides of the polygons are bent inwards slightly. If we therefore allow the sides to bend elastically, the defects at the vertices may be removed by bending [Fig. 5(a)], allowing the growth of protrusions.

The energetics are particularly simple for triangles: bending each of the sides of an equilateral triangle of side length $\ell$ into a circular segment of length $\ell$ intercepting an angle of $60^{\circ}$ on a circle of radius $3 \ell / \pi$ removes the defects at the corners [Fig. 5(a)] and reduces the surface area by an amount $\sim \ell^{2}$ at a bending energy cost scaling as $\ell(1 / \ell)^{2} \sim 1 / \ell$. Hence, if $\ell$ (or, equivalently, $\mathcal{P}$ ) is large enough, the disappearance of the defects is energetically favorable. The same criterion applies, perhaps counterintuitively, to parallelograms or trapezoids [14]. It is, however, clear that more bending is required to remove a defect of $120^{\circ}$ than one of $60^{\circ}$, and, hence, we expect protrusions to grow only from the acute angles of parallelograms or trapezoids, as observed [Fig. 5(b)].

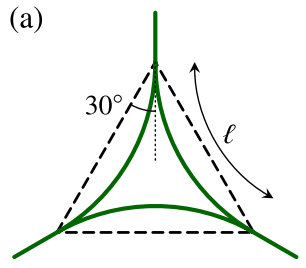

(b)

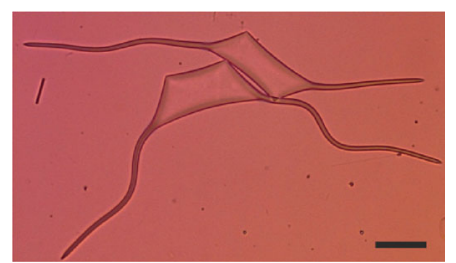

FIG. 5. Growth of protrusions. (a) Geometry: bending of sides removes defects and allows protrusions to grow. (b) Parallelograms and trapezoids sprout protrusions from $60^{\circ}$ angles, but not from $120^{\circ}$ angles. Scale bar $20 \mu \mathrm{m}$. 
In the simplest model, protrusions either grow indefinitely or do not grow at all: consider a parallelogram with two equal protrusions, each of nondimensional length $p$. Let $\alpha_{p}$ denote the characteristic nondimensional width of the protrusions. In line with our previous assumptions, we assume $\alpha_{p}$ to depend on the material properties only. The energy is thus

$\mathcal{E}=2\left[\mathcal{A}+\alpha_{p}(2 p)\right]-\alpha[\mathcal{P}+2(2 p)]=\mathcal{E}_{0}+4\left(\alpha_{p}-\alpha\right) p$,

where $\mathcal{E}_{0}=2 \mathcal{A}-\alpha \mathcal{P}$ is the energy in the absence of protrusions. In particular, if $\alpha>\alpha_{p}$, protrusions grow ad infinitum and do not grow at all otherwise. We roughly estimate $\alpha_{p} \sim H / P_{0}$, and thus $\alpha / \alpha_{p} \sim A_{r} \Delta \mu / \gamma H \sim 18$, and, hence, we do expect to see the formation of protrusions in experiments. These experiments reveal, however, that protrusions in parallelograms may shrink and disappear again as the parallelogram elongates into a thin rod. This effect results from volume conservation and the three-dimensional structure of the droplet [Fig. 4(b)]: after the curvature of the droplet has changed sign, shrinking the protrusions reduces the surface area (both of the protrusions and of the bulk of the droplet, because volume conservation means that its surface curves inwards less), but does incur a cost of reducing $\mathcal{P}$. This competition may cause the protrusions to shrink; in particular, the surface area reduction is larger the smaller is $\mathcal{A}$ (which is the reason why this effect is observed in elongated parallelograms rather than triangles, for instance). This effect is further enhanced, at the same $\mathcal{A}$, for more elongated parallelograms [14].

In this Letter, we have shown how the simplest competition between a single phase transition and surface tension in a discrete geometry affords a wealth of shape transitions. Based on the experimental observations, the model reproduces and explains the key experimental findings of Refs. [3,7]: the sequence of shapes with the observed dichotomy between triangles and quadrilaterals, the alternative outcomes of puncturing quadrilaterals or elongating rods, and the formation and dynamics of protrusions exuding from the vertices of the platelets. Taking the analysis from the plane to three dimensions and understanding the geometric basis for the flattening of the droplets is a key challenge for future work, as are the kinetics of the phase change driving the shape evolution.

We thank S. Tcholakova and I. Lesov for discussions and experimental data. P. A. H. and S. K. S. are grateful to the Department of Chemical and Pharmaceutical Engineering of the University of Sofia for their hospitality during the annual retreat of the department at Giolechitsa (Bulgaria). This work was supported in part by the Engineering and Physical Sciences Research Council (P. A. H.), an Established Career Fellowship from the EPSRC (R.E. G.), and the European Research Council (Grant EMATTER No. 280078 to S. K. S.).
*R.E.Goldstein@damtp.cam.ac.uk †sks46@cam.ac.uk

[1] A. M. Turing, The chemical basis of morphogenesis, Phil. Trans. R. Soc. B 237, 37 (1952).

[2] Plato, Пoגıєєí (The Republic) (Ancient Greece). See, e.g., http://classics.mit.edu/Plato/republic.mb.txt

[3] N. Denkov, S. Tcholakova, I. Lesov, D. Cholakova, and S. K. Smoukov, Self-shaping of oil droplets via the formation of intermediate rotator phases upon cooling, Nature (London) 528, 392 (2015).

[4] E. B. Sirota and A. B. Herhold, Transient phase-induced nucleation, Science 283, 529 (1999).

[5] S. Guttman, Z. Sapir, M. Schulz, A. V. Butenko, B. M. Ocko, M. Deutsch, and E. Sloutskin, How faceted liquid droplets grow tails, Proc. Natl. Acad. Sci. U.S.A. 113, 493 (2016).

[6] S. Guttman, B. M. Ocko, M. Deutsch, and E. Sloutskin, From faceted vesicles to liquid icosahedra: Where topology and crystallography meet, Curr. Opin. Colloid Interface Sci. 22, 35 (2016).

[7] D. Cholakova, N. Denkov, S. Tcholakova, I. Lesov, and S. K. Smoukov, Control of drop shape transformations in cooled emulsions, Adv. Colloid Interface Sci. 235, 90 (2016).

[8] N. Denkov, D. Cholakova, S. Tcholakova, and S. K. Smoukov, On the mechanism of drop "self-shaping" in cooled emulsions, Langmuir 32, 7985 (2016).

[9] M. J. Bowick and R. Sknepnek, Pathways to faceting of vesicles, Soft Matter 9, 8088 (2013).

[10] J. Lidmar, L. Mirny, and D. R. Nelson, Virus shapes and buckling transitions in spherical shells, Phys. Rev. E 68, 051910 (2003).

[11] This assumes that the coefficients of surface tension between the water, on the one hand, and the liquid or rotator phases, on the other, are equal, and ignores the surface tension between the two oil phases (or, alternatively, that the interfacial tension between the water and the liquid phase is approximately equal to the sum of the interfacial tensions between the water and rotator phases and the rotator and liquid phases). These additional effects would but modify slightly the final parameter, as would taking into account the density difference between the two phases.

[12] H. Kraack, M. Deutsch, and E. B. Sirota, n-alkane homogeneous nucleation: Crossover to polymer behavior, Macromolecules 33, 6174 (2000).

[13] Z. Suo, Motions of microscopic surfaces in materials, Adv. Appl. Mech. 33, 193 (1997).

[14] See Supplemental Material at http://link.aps.org/ supplemental/10.1103/PhysRevLett.118.088001, which includes Ref. [15], for a derivation of the governing equations, a detailed discussion of the three-dimensional effects, and videos of simulations of evolving droplets.

[15] W. Helfrich, Elastic properties of lipid bilayers: Theory and possible experiments, Z. Naturforsch. C 28, 693 (1973).

[16] This assumes symmetric defects; one could, however, conceive of protrusions that are aligned with one of the edges at the vertex whence they sprout. Such protrusions are observed but rarely, suggesting that these "composite" defects are energetically unfavorable, too. 